\title{
Flying with an Umbrella: Operational Strategies for the Tandem-L Mission
}

\author{
Matthias Dauth ${ }^{1}$, Daniel Schulze ${ }^{2}$, Markus Bachmann ${ }^{3}$, Daniela Borla Tridon ${ }^{4}$, Ralph Kahle ${ }^{5}$, and Edith Maurer ${ }^{6}$ \\ German Space Operations Center (GSOC), German Aerospace Center (DLR), D-82234 Wessling, Germany
}

Tandem-L is a highly innovative satellite mission consisting of two identical satellites, which shall fly in a close formation in low earth orbit. The mission's goal is to monitor a wealth of dynamical parameters in the main realms of our environment: The Biosphere, Geosphere, Cryosphere, and Hydrosphere. Key to the mission is a novel design of the radar instrument, which operates in L-band and includes a large deployable reflector. On the one hand the design incorporating the reflector leads to considerable improvements in terms of sensitivity of the instrument and of spatial resolution. On the other hand it raises the bar for the satellite architecture and mission operations significantly. This paper gives an overview of the planned mission, illustrates the distinctive features of the twin Tandem-L satellites, and focuses on requirements on the attitude and orbit control system. It further presents operational strategies, which are in large parts derived from flight heritage of TerraSAR-X and TanDEM-X at the German Space Operation Center.

\section{Nomenclature}

$\begin{array}{ll}\text { GSOC } & =\text { German Space Operations Center } \\ \text { TDL } & =\text { Tandem-L (mission) } \\ \text { TL1 } & =\text { Tandem-L satellite } 1 \\ \text { TL2 } & =\text { Tandem-L satellite } 2 \\ \text { LEO } & =\text { Low Earth Orbit } \\ \text { TDX } & =\text { TanDEM-X (satellite) } \\ \text { TSX } & =\text { TerraSAR-X (satellite) } \\ \text { AOCS } & =\text { Attitude and Orbit Control System } \\ \text { MOS } & =\text { Mission Operations System } \\ \text { FDS } & =\text { Flight Dynamics System } \\ \text { MPS } & =\text { Mission Planning System }\end{array}$

\section{The Tandem-L Mission}

\begin{abstract}
Climinat limate change is one of the most pressing issues our and next generations have to face. To develop strategies that are able to mitigate global warming and its potentially severe impact on the environment, a deep understanding of the ongoing processes and their dynamics is essential. Yet, refining currently used climate models requires a broad set of reliable data over various time scales, which is often missing. Prompted by the urgent need for information, the German Aerospace Center (DLR) and an alliance of scientists from participating Helmholtz institutes (Helmholtz alliance 'Remote Sensing and Earth System Dynamics') have proposed the Tandem-L mission [1] [2]. It is a highly innovative radar mission dedicated to globally monitor the four major realms of our environment: the Biosphere, Geosphere, Cryosphere, and Hydrosphere. Its specific tasks are illustrated in Fig. 1. They range from mapping of forest biomass and its changes, monitoring of deformations at volcanoes and of geologically active regions in general, generating digital elevation models, to performing acquisitions of soil moisture, sea ice extent, or glacier and ice cap dynamics. All these objectives, which underpin the versatility of Tandem-L, will make vital contributions to various fields of research. To name but a few, the global measurement of

${ }^{1}$ GSOC TDL AOCS Engineer, GSOC Mission Operations, Matthias.Dauth@dlr.de

${ }^{2}$ GSOC TDL Lead System Engineer, GSOC Mission Operations, Daniel.Schulze@dlr.de

${ }^{3}$ DLR-HR TDL Ground Segment Project Manager, Microwave and Radar Institute, Markus.Bachmann@dlr.de

${ }^{4}$ DLR-HR TDL System \& Performance Engineer, Microwave and Radar Institute, Daniela.BorlaTridon@dlr.de

${ }^{5}$ GSOC TDL Flight Dynamics Lead, GSOC Space Flight Technology, Ralph.Kahle@dlr.de

${ }^{6}$ GSOC TDL Mission Lead, GSOC Mission Operations, Edith.Maurer@dlr.de
\end{abstract}


forest biomass and its temporal variation is important to understand the carbon cycle. Geoscience and earthquake risk analysis can benefit from deformation maps that Tandem-L will provide with millimeter accuracy. Further, monitoring the movement of glaciers and their melting process will help to predict sea level rises.

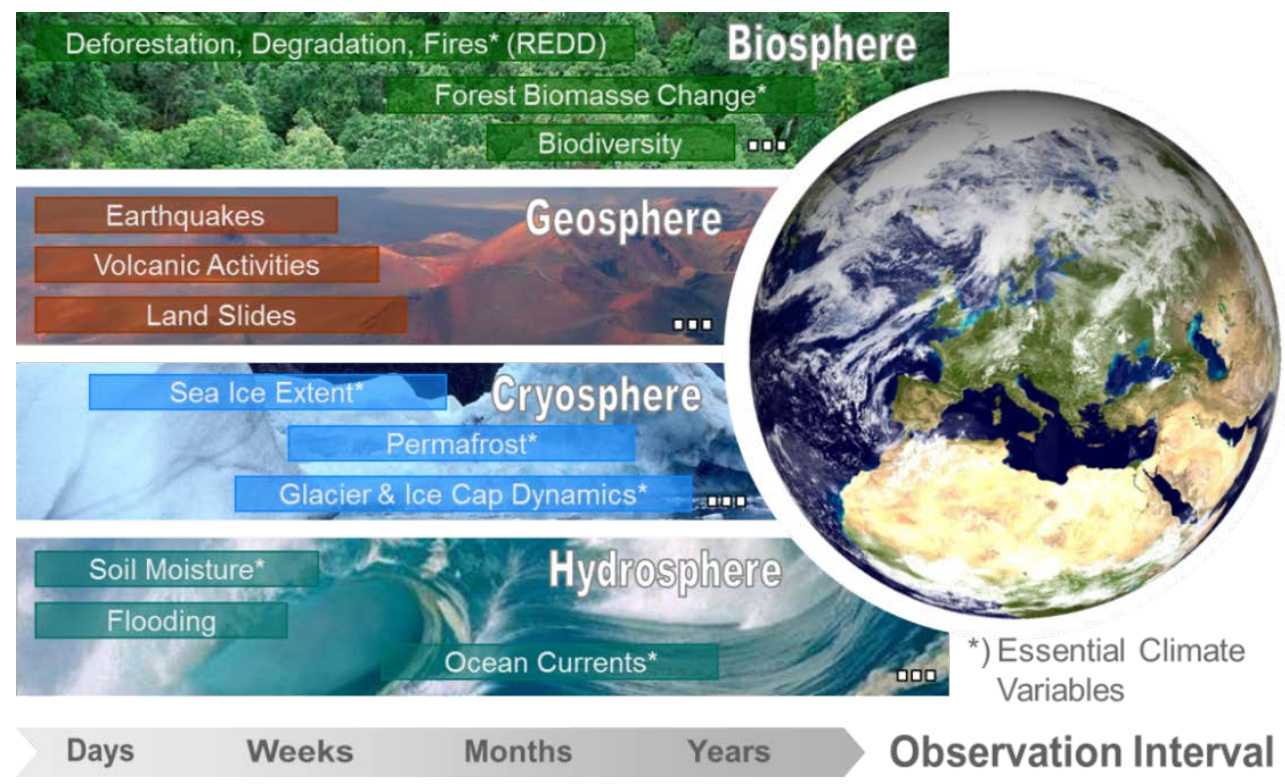

Figure 1: Illustration of Tandem-L's mission objectives.

Two identical satellites (TL1 and TL2, which are illustrated in Fig. 2) lay the foundation of the mission. They are planned to be launched around 2024, while the project is currently in the phase of requirement definition (ECSS B1). The twins will be placed in low earth orbit (LEO) at an altitude of $745 \mathrm{~km}$ in a dual launch. Similar to their predecessor mission, TanDEM-X [3], both Tandem-L satellites will fly in a closely controlled formation to permit single-pass radar interferometry. The formation will be adjusted during different phases of the mission to best suite the observation plan. For bi-static radar acquisition, which is used, e.g., to generate tomographic images or to process a digital elevation model, the inter-satellite distance can decrease down to $400 \mathrm{~m}$ during nominal radar operations.

The eye-catching feature of the satellites certainly is the large deployable reflector (LDR). In deployed configuration the metallic mesh structure mounted in zenith direction not only dominates the appearance of the satellites but is an essential part of the Synthetic Aperture Radar (SAR) instrument [2]. Specifications of the LDR are a diameter of $15 \mathrm{~m}$ and a concave curvature with a focal length of $13.5 \mathrm{~m}$. It redirects the radar beams emitted by the active elements of the space-pointing instrument towards earth. On the reversed path the instrument receives the radar echoes through reflection at the LDR. To play to its full strengths, the instrument's design combines the LDR with a two-dimensional digital feed array. This permits a flexible beam shaping on transmit with optimum swath illumination. On receive the radar echo gets quantized as it is ingested into specific elements of the feed array. As each element of the feed array receives echo signals from non-overlapping angular domains, a direct mapping between the feed array's topology and the earth's surface is possible. Since Tandem- $\mathrm{L}$ is the first radar mission to fly with a fully digital setup, the satellites are equipped with various on-board processing and filtering techniques and feature sophisticated operational concepts such as scan-on-receive and staggered SAR [2] [4] [5]. Overall, the novel instrument design lifts current limitations on signal strength, resolution, swath widths, and time in actual service. Furthermore, different operational modes can be tailored to different scenarios. Operating in fully polarimetric mode, for example, swath widths of $175 \mathrm{~km}$ with a resolution better than $7 \mathrm{~m}$ are possible. When switching to single-pol mode even a swath width of $350 \mathrm{~km}$ can be acquired [2]. As the instrument can be operated almost continuously, a weekly global coverage is possible, which makes the mission especially suited for measuring dynamic processes on earth's surface. 


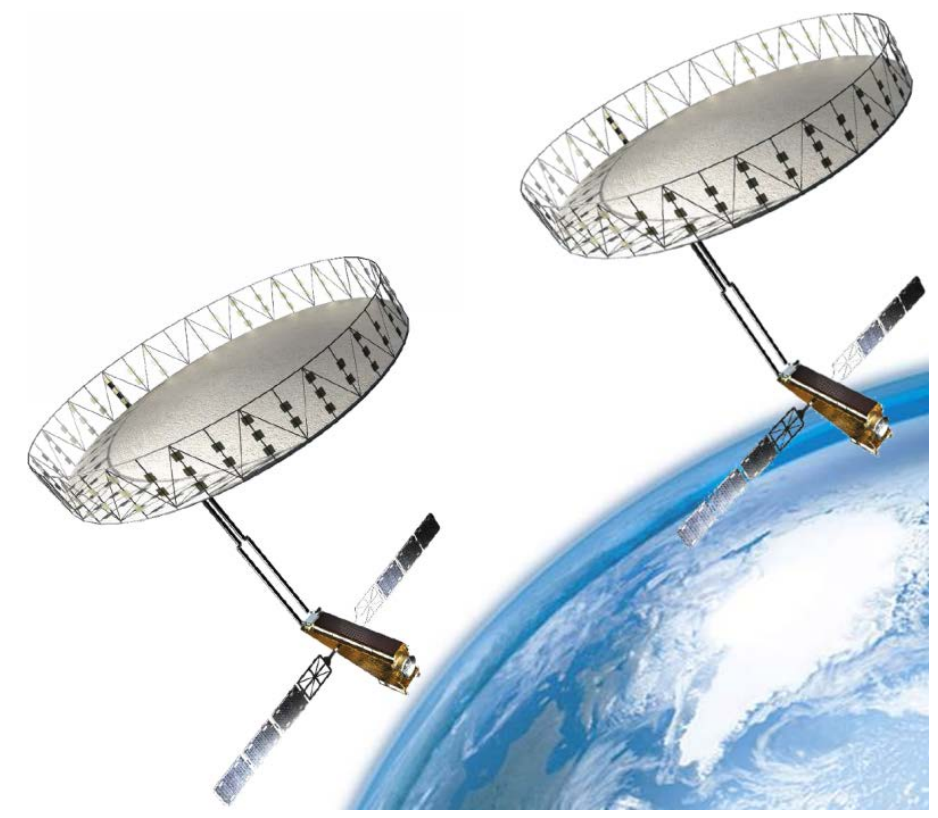

Figure 2: Artist's impression of the twin Tandem-L satellites with the deployed reflector. In nominal operations, the radar instrument (dark panel on top of the satellite body) is oriented in zenith direction. In right-looking attitude, the solar panels are illuminated from behind and they lie in the orbital plane. The flight direction is oriented to the upper right (parallel to the solar panels).

As the mission's name suggests, the SAR instrument of Tandem-L operates in L-band. This brings two key advantages compared to the typically used shorter wavelength of X- or C-band. First, a greater penetration depth of volume scatterers allows for scanning through thick vegetation and thus for generating tomographic images of biomass or glaciers. Second, the improved temporal coherence permits for large scale surveying of deformations with millimeter accuracy with repeat-pass interferometry.

On the one hand all these facts underline the immense potential that Tandem-L offers. On the other hand they pose challenges for mission operations.

\section{Mission operations concepts}

In the following chapters we will dive deeper into the details of the mission from an operational perspective. Paragraph A gives a short overview of the general mission operations concept. Paragraphs B, C, and D deal with specific challenges of the mission.

\section{A. General operations concept}

Due to the mission's high complexity, all tasks for conducting mission operations successfully are distributed to three parties within the ground segment. Tasks related to the radar instrument, such as SAR calibration, SAR payload operations, and formation geometry and acquisition planning, lie within the responsibility of the Instrument Operations and Calibration System (IOCS). As the acquisition strategy leads to an immense data volume of up to eight terabytes per day, the Payload Data System (PDS), which is the second party, is required to provide enough downlink capacity as well as the capacity to archive and process the data. For the downlink, the PDS will establish a dense ground station network of Ka-band antennas. Although Ka-band technology is currently under development for LEO satellites, it is selected as the baseline for the mission as it achieves an average data rate above two Gbit per second. While Tandem-L uses Ka-band for the payload data, telecommands and housekeeping telemetry are transmitted via S-band. The Mission Operations System (MOS) is responsible for providing the necessary infrastructure to command the spacecraft and to monitor the spacecraft's health. Moreover, MOS will implement a fully automated Mission Planning System (MPS) which shall generate conflict-free and consistent timelines for commanding the satellites. This includes the scheduling of uplink and downlink activities for S- and Ka-band, execution of SAR data acquisitions, and other nominal spacecraft operations. The MPS further implements a priority 
concept and considers all known spacecraft as well as ground system constraints, e.g., instrument restrictions, formation constraints, on-board memory limits, and ground station availabilities. To achieve these tasks, the MPS provides and supports automated input and output interfaces to all relevant ground segment subsystems. Specifically for the Tandem-L mission, communication with the IOCS, which issues the acquisition and formation plan, is necessary to compose the actual acquisition sequences for both satellites. Here, the focus of the MPS lies in the implementation of a short-term mission timeline. An excerpt of this timeline is exported as command timelines during every timeline uplink session. The currently developed generic reactive planning framework [6] will be employed to react quickly to changes of boundary conditions and thus to adapt the mission timeline on short notice.

\section{B. Formation and Constellation Flight}

A major challenge of the TDL mission is the planning and realization of the desired orbit and formation geometries. In general, one of the two satellites will fly on a predefined reference orbit (master), while the other (slave) will fly on a slightly different orbit. The slave's orbit depends on the current formation. The master satellite will operate in a sun-synchronous dusk-dawn orbit, which is particularly suited for continuous radar observations due to steady illumination of the solar panels. Thus, it guarantees enough power except for short eclipse phases around summer solstice, in which the load lies entirely on the battery. The nearly circular target orbit has an altitude of $745 \mathrm{~km}$, an inclination of $98.38^{\circ}$, and an 18:00 mean local solar time at ascending node. Further, the orbit repeats itself in a 16 days cycle and is defined with respect to an earth-fixed reference [7]. The repeat cycle allows to revisit targets within a short time interval and thus to monitor deformations on short time scales. This also brings the benefit of having fast access to targets in case of emergency observations. To maintain the osculating orbit within $250 \mathrm{~m}$ crosstrack distance from the reference orbit, the well-established TerraSAR-X orbit control concept will be implemented [8]. Thereby, weekly to monthly drag make-up manoeuvers depending on the solar activity and about four inclination control maneuvers per year will be required to maintain the master satellite within the control tube.

Yet, orchestrating the formation of two spacecraft flying in close vicinity increases the challenge significantly. During the whole mission, the formation will be adapted to best serve the observation scenario. In principle, the mission foresees three different flight configurations: a close formation, a pursuit formation, and a constellation phase. The design of the exact formation parameters is driven by interferometry requirements, especially the height of ambiguity [9] [10]. The latter is proportional to the inverse of the orthogonal baseline, i.e., the cross-track distance of the antenna phase centers between both satellites orthogonal to the line of sight. Target formation parameters are designed in order to vary the orthogonal baseline from a few hundred meters to almost twenty kilometers. Figure 4 illustrates the formation phases for a reference scenario of two years depicting the evolution of horizontal (top) and along-track (bottom) separation. The reference scenario repeats every two years throughout the 12 year mission duration.

For implementing the formation flight, the GSOC team benefits from a long history of operational experience. In particular, experience from TanDEM-X can be transferred to Tandem-L since the satellites will orbit each other in a similar fashion during the close formation phase [11]. Here, the master satellite is controlled with respect to the reference orbit as described above. The slave satellite flies on an almost identical trajectory but with small differences in eccentricity, in the right ascension of the ascending node (RAAN) as well as in inclination. These differences yield a helix-shaped relative motion of the twin satellites, which is illustrated in Fig. 3. In general, the horizontal baseline is at its maximum at the equator crossings due to the RAAN offset and decreases with higher latitudes. Around the poles the horizontal baseline vanishes and a vertical separation is achieved by the differences in the orbits' eccentricities. At the opposing poles the formation is therefore inverted, i.e., TL1 is beneath TL2 at one pole and reversely at the other. In order to minimize the fuel expenditure for realization of the very large and also frequent changes in horizontal separation, small inclination offsets are installed. Thereby, perturbations arising from earth's oblateness force the master's and slave's RAAN to alter differently. For example, a maximum inclination offset of $0.0137^{\circ}$ will be installed in order to yield a horizontal drift rate of $3200 \mathrm{~m}$ per 16-day repeat cycle (cf. Figure 4: first quarter of 2023). Overall, the mission requires the baseline between both satellites to be controlled 
with an accuracy of $50 \mathrm{~m}$ (3-sigma, 1-D) in radial and normal direction and $300 \mathrm{~m}$ (3-sigma, 1-D) in along-track direction. To control the formation, we anticipate inclination control maneuvers every 16 days and daily maneuvers to maintain the relative eccentricity and along-track separation.

While the close formation phase is ideal for measuring, e.g., forest heights or generating a digital elevation model, it lacks the required baseline at high latitudes for ice structure acquisitions. The second type of formation is designed to fill that gap. In the so-called monostatic pursuit formation one spacecraft directly follows the other with an alongtrack distance of several hundred kilometers. Apart from TanDEM-X [12], we can also build on the expertise gained from the GRACE mission, where the along-track distance between both satellites was about $220 \mathrm{~km}$ with control bands at $\pm 50 \mathrm{~km}$ [13]. Moreover, a similar constellation is planned for its successor Grace-FollowOn [14]. In case of Tandem-L's pursuit formation phase, the time offset due to the along-track distance and the corresponding rotation of the earth provides sufficiently large baselines at high latitudes. In contrast to the close formation, in which the data acquisition is performed in bistatic mode, the pursuit phase is based on monostatic acquisitions. In the latter transmitter and receiver are collocated and both satellites acquire data independently, which effectively doubles the baseline.

During the third formation phase, i.e., the constellation phase, both spacecraft are controlled against the same reference ground track. However, they are displaced in along-track direction by about $2800 \mathrm{~km}$ or even $5600 \mathrm{~km}$, to effectively reduce the combined revisit times of a target to an integer number of days, i.e., $7+9$ or $2+14$ days, respectively. Especially applications which require frequent interferometric observations like agriculture or soil moisture measurements benefit from the constellation mode. It may also be very useful for tailoring the data acquisition in accordance with the needs following earthquakes or other natural disasters.

Aside from the formation, also the transitions between them are optimized by the GSOC flight dynamics team. An optimized formation schedule for a two years cycle, see Figure 4, was established which incorporates the long term acquisition plan with a minimum required delta-v. During the mission, this cycle will be repeated six times.

In addition to the three main phases, a fourth operational configuration is foreseen. Most of the time, the attitude of the satellites is chosen such that the footprint of the radar beam lies on the right side of the orbit plane. This and the geometry of the orbit (especially its inclination) make it difficult to comprehensively observe areas in the Antarctica. To lift this restriction, both satellites perform $180^{\circ}$ slews about their yaw axis to be able to operate in the so-called left-looking mode within dedicated phases of the mission.

Besides the advanced formation control, also a precise determination of the orbit and the interferometric baseline is required. For precise orbit determination the flight dynamics team will post-process GNSS data to achieve an absolute position accuracy of $10 \mathrm{~cm}$ or better (1-sigma, 1-D) in any direction orthogonal to the velocity vector. To determine the cross-track baseline, which is defined by the distance of the phase centers of the radar instrument, with the required accuracy of $8 \mathrm{~mm}$ (1-sigma) more effects than the orbit precision have to be considered. Attitude errors have to be kept as small as possible including vibrations or deformations of the LDR. Further, uncertainties may arise from variations of the center of mass, where fuel sloshing can be a decisive contribution to the mass distribution.

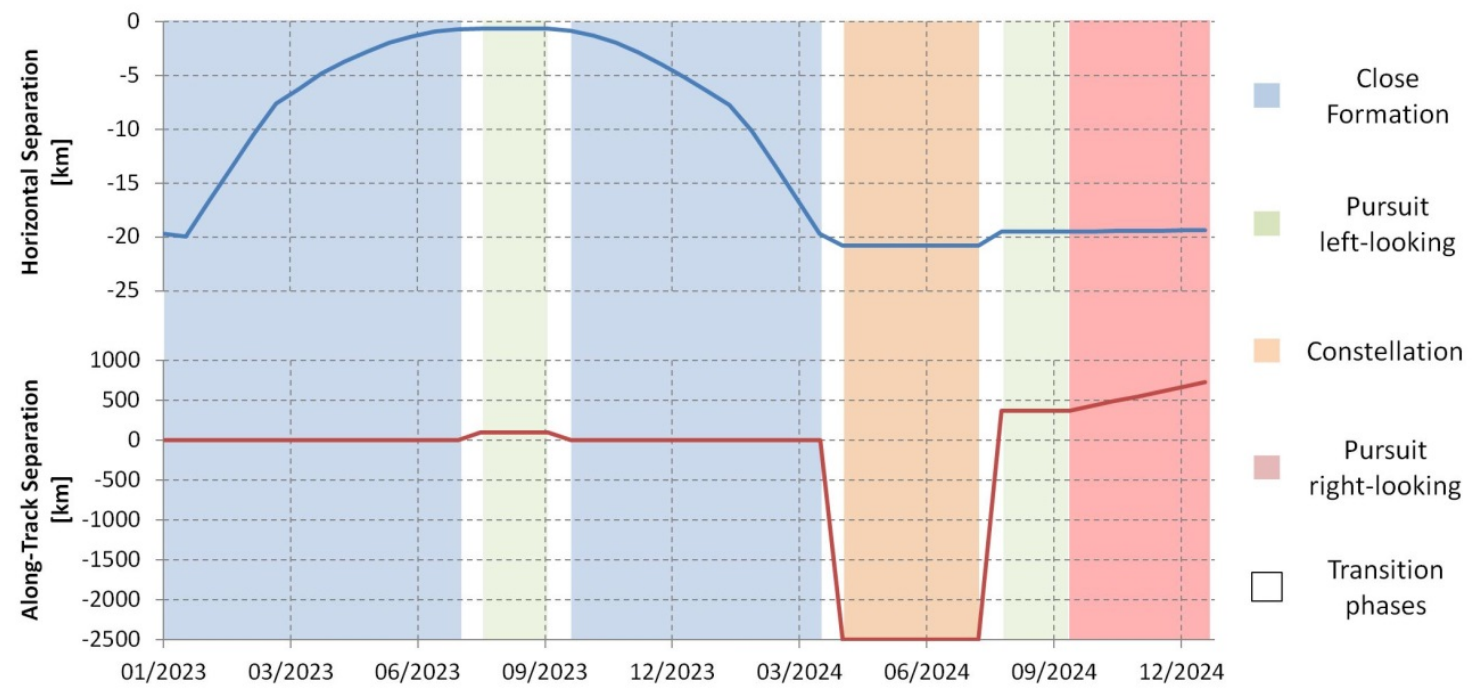

Figure 4: Evolution of the formation within a two years cycle. The top panel shows the horizontal separation of the two spacecraft, the lower panel the along-track separation. Different mission

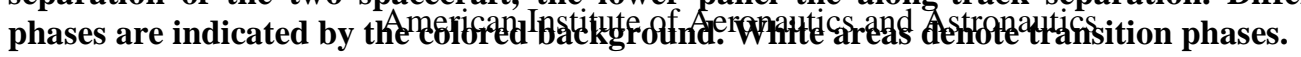




\section{Safety aspects and a comparison to TanDEM-X}

A big challenge for operations is to ensure the safety of the two satellites while flying with approximately $7 \mathrm{~km} / \mathrm{s}$ at a separation of only $400 \mathrm{~m}$. Here, the flight proven safety measures implemented for TanDEM-X [15] [11] can serve as a guideline for Tandem-L. In the following we will contrast both missions and explicitly state differences in their concepts to work out suitable measures for Tandem-L.

In general, the parameterization of the formation is designed to avoid collision. As described in detail in Ref. [16], the inclination and eccentricity vector separation makes the formation passively stable. However, for TSX and TDX, additional measures were required. The initial AOCS safe mode concept of TSX solely used a thruster based reaction control system. The problems for the safety of the formation arise from the accommodation and orientation of the thrusters: As all $4+4$ (nominal + redundant) thrusters are located at the back panel of the satellite and their orientation is only slightly tilted, a control torque is always accompanied by parasitic forces. The latter can alter the orbit significantly in an unpredictable way and as a consequence could lead to a higher collision probability. With the launch of TDX, which was three years after the launch of TSX, the AOCS safe mode concept had to be reworked and a new magnet-torquer based safe mode was introduced [15]. Furthermore, a dedicated on-ground procedure was established that summarizes all necessary actions in case of failures that pose a threat to the formation's safety. To avoid a similar threat, the Tandem-L satellites are required to have a completely force free attitude control for all AOCS modes. This requirement was further extended to also guarantee torque-free forces for orbit maneuvers.

Apart from the collision probability also mutual illumination by transmitted high-power radar signals can damage the satellites' electronics. To cope with these risks, multiple safety measures have been established for TSX/TDX. The primary mechanism to protect against illumination is the so-called exclusion zones. Within the angular interval in which one spacecraft would pass through the radar beam of the other satellite, radar operations are prohibited. There are several instances of exclusion zone checks. First, each of the partner satellites obeys the exclusion angles autonomously. However, the angles at which on-board control prohibits radar activities have to be set by command. Consequently, their configuration has to be adapted each time the formation is changed. In addition to the on-board exclusion zones, another instance is established on ground. Here, all constraints imposed by the exclusion zones are already considered by mission planning in the generation of the mission timeline, which contains all commands for radar operations. These limits are chosen even more conservative than their on-board counterparts. Finally, before uploading a mission timeline, a final check is performed within the command system. As an additional measure, Flight Dynamics prepares transmission exclusion windows with each maneuver. All these checks assume that both satellites fly in their anticipated orbit. Therefore, safety cannot be guaranteed by the exclusion zones alone. To cover such situations, the satellites are protected by additional mechanisms which relay on information exchange in flight. Sync-warning data takes transfer, for example, time-synchronization signals bi-directionally. The sync-warnings are planned by the MPS on a regular basis, usually every 53 minutes, and must be performed successfully before actual data takes. To do so, dedicated sync horns mounted on both satellites are transmitting a signal. Each of the sync horns emits in a specific direction and a spherical coverage is in principle possible. The crucial point here is that only those sync horns are selected which emit in the direction where the other satellite is supposed to be. If a sync warning fails, i.e., the satellites do not receive the anticipated signal because of orbit or attitude deviations, the instruments get passivated.

The Inter-Satellite Link is the second in-orbit communication line. It uses the S-band equipment to transfers basic AOCS, GNSS, and satellite mode information from TSX to TDX. In case of an AOCS safe mode on TSX, the instrument of TDX gets passivated immediately. Yet, the main intention of the Inter-Satellite Link was to facilitate TDX with all necessary information for autonomous formation control. During the TDX autonomous formation flight (TAFF) campaigns, TDX planned and performed the formation keeping manoeuvers without ground interaction. TAFF was especially tailored to meet the high control accuracy requirement (along-track separation of $50 \pm 10 \mathrm{~m}$ ) for along-track interferometry used for measuring ocean currents [17]. As the demands on TDL are relaxed compared to the TAFF campaign on TanDEM-X, manoeuvers are planned on ground only.

For Tandem-L the illumination risk is partly mitigated but has a different manifestation. Both Tandem-L satellites are designed in such a way that they can withstand mutual illumination if their distance is larger than $400 \mathrm{~m}$. Therefore, strict mission requirements have been established. During nominal radar operations, the inter-satellite distance shall not fall below the $400 \mathrm{~m}$ threshold. Only during periods of SAR outage on both satellites, i.e., maintenance phases for formation acquisition or formation reconfiguration, the minimum distance between the satellites is allowed to be decreased to $150 \mathrm{~m}$. For an arbitrary satellite attitude the requirement translates into a $200 \mathrm{~m}$ minimum distance. To ensure that active radar transmission is not commanded in this scenario, a series of onground checks will be implemented at GSOC. They will explicitly check whether the mission timeline is compatible 
with radar operations, considering formation configurations and manoeuvers. Several options are currently discussed. A viable strategy could be that mission planning generates the mission timelines including all manoeuvers commands exclusively. Therefore, the location and time of each manoeuver has to be consolidated between MPS and FDS beforehand. For all maneuvers a risk assessment and a statement whether the $400 \mathrm{~m}$ requirement might be violated has to be delivered by FDS. The risk assessment would have to include possible failure scenarios, such as manoeuvers being executed on one satellite only or on neither of both. If the $400 \mathrm{~m}$ requirement is endangered, no radar commands are allowed to be inserted into the timeline. Within an alternative approach, the timeline and maneuver commands would have to be consolidated. Yet, both are exported to the command system by FDS and MPS separately. To verify their compatibility, the timeline as well as the maneuver commands carry unique identifiers which have to compatible. In case of a mismatch, a warning has to be issued and the release of commands is prohibited. Besides the on ground checks, the current baseline does not foresee an equivalent concept to the Inter-Satellite Link or the sync warning data takes of TDX and TSX.

To react quickly in case of problems on-ground and on-board, the well-established on-call strategy of the TanDEM$\mathrm{X}$ mission was proven to be successful [18]. This involves that all ground contacts are monitored round-the-clock by the multimission flight support. In case of anomalies, one engineer of each subsystem is reachable outside usual office hours and has the duty to be at the control center within one hour. Further, the engineers can follow incoming telemetry in real-time, even outside the control center by means of Satmon@home [19].

The on-call strategy also extends to the Flight Dynamics team as debris collision avoidance will play an important role in routine operations as the large spacecraft structure is exposed to a densely populated orbit region. If the collision probability increases to values that are not tolerable, i.e., to probabilities higher than $10^{-4}$, additional maneuvers can be exported. In an ideal scenario the standard formation keeping maneuvers can be adapted with a shift in execution time or change in delta-v.

\section{Tandem-L's prominent feature: The LDR and its effect on the AOCS}

All data acquisitions have to be performed with unprecedented accuracy to achieve the mission's goals. Part of that boils down to the pointing performance of the radar instrument. Orienting the spacecraft in the right direction and keeping it in a stable position is challenging, given the huge LDR structure. Technically, the whole satellite has to be able to achieve a pointing accuracy of the radar antenna beam during all nominal SAR operations (1 sigma confidence level) of $\pm 0.04^{\circ}$ in elevation and azimuth. The pointing stability, expressed as the maximum pointing error variation over a time interval of one second, shall not exceed $\pm 0.01 \%$ sec in elevation and in azimuth (1 sigma confidence level). However, the thresholds for the on-ground knowledge of the beam pointing performance is even stricter with $\pm 0.02^{\circ}$ in both directions, in order to achieve the precision necessary for processing the SAR products. All this calls for a sophisticated AOCS. The sensors equipment will be based on GNSS receivers, star trackers, sun sensors, and magnetometers. As the parts of the upper hemisphere are blocked by the LDR, the accommodation of the sensors is critical. Apart from optical sensors or infrared sensors, the metallic mesh structure of the LDR also shields GNSS signals which have similar frequencies to the radar instrument.

The attitude will be controlled, depending on the AOCS mode, by a force free thruster system, magnetorquers, and reaction wheels. During nominal operations a fine pointing AOCS mode, which will employ reaction wheels and magnetorquers, has to keep the satellite in nadir pointing direction. Since the reflector structure introduces large offdiagonal elements in the moments of inertia tensor, gravity gradient and gyroscopic torque, which are in the order of tenths of $\mathrm{mNm}$, have to be counterbalanced continuously to keep a stable attitude. Considering nadir pointing as the initial condition, the satellite would oscillate about its roll axis by several degrees if no active compensation is applied. Although the large surface area of the LDR structure is oriented perpendicular to the flight direction, also aerodynamic torque has to be compensated by the reaction wheels.

Similarly to TSX and TDX the AOCS of Tandem-L has to support a total zero-Doppler steering, which mainly turns the satellite about its yaw axis by a maximum of $4^{\circ}$ depending on the argument of latitude. Fundamentally different form the concept of TSX/TDX is the left-looking mode of Tandem-L. To reach the left looking attitude with TSX/TDX, the spacecraft slews about the roll axis by $67^{\circ}$. Since roll has the smallest principal moment of inertia, the slew takes only a few minutes. Due to the short times, left looking data takes are scheduled on a regular basis, typically 1-3 per day. In contrast, Tandem-L turns about the yaw axis by $180^{\circ}$, which has considerably bigger moment of inertia. Depending on which actuators perform the slew, we expect transition times up to 45 minutes. Therefore, Tandem-L only acquires data in left-looking attitude during dedicated mission phases. 


\section{Conclusion}

Overall, Tandem- $\mathrm{L}$ is an extremely ambitious mission that has the potential to open new avenues for a broad area of research. The mission's focus lies on a global observation of dynamic processes on the Earth's surface with unprecedented quality and resolution. All this calls for a large variety of innovative solutions within the space and ground segments. These range from the novel design of the radar instrument, an advanced satellite bus, and powerful data processing techniques, to advanced operational strategies. For the latter we presented an overview of the mission's operational concept. To adapt the formation to different types of acquisitions, different formation phases were introduced. We presented strategies to maintain the formation within the required limits. To ensure the safety of both satellites we drew a comparison between the well-established measures used for the TanDEM-X mission and the requirements of Tandem-L preventing collisions and mutual illumination. Finally, we illustrated specific requirements on the AOCS mainly imposed by the LDR structure and the beam pointing accuracy.

\section{Acknowledgments}

The work described here was carried out as part of the DLR projects TerraSAR-X, TanDEM-X, and Tandem-L.

\section{Bibliography}

[1] Tandem-L brochure, Mai 2017. [Online]. Available: http://www.dlr.de/hr/en/Portaldata/32/Resources/dokumente/tdml/Tandem-L_Brochure_2017-05.pdf.

[2] A. Moreira, G. Krieger, I. Hajnsek, K. Papathanassiou, M. Younis, P. Lopez-Dekker, S. Huber, M. Villano, M. Pardini, M. Eineder, F. DeZan, and A. Parizzi, "Tandem-L: A Highly Innovative Bistatic SAR Mission for Global Observation of Dynamic Processes on the Earth's Surface," IEEE Geoscience and remote sensing magazine, pp. 9-23, 2015.

[3] TanDEM-X brochure, 2010. [Online]. Available: http://www.dlr.de/hr/en/Portaldata/32/Resources/dokumente/broschueren/TanDEM-X_web_Brochure2010.pdf.

[4] F. Bordoni, M. Younis, E. M. Varona, G. Krieger, "Adaptive scan-on-receive based on spatial spectral estimation for high-resolution, wide-swath Synthetic Aperture Radar," Geoscience and Remote Sensing Symposium,2009 IEEE International,IGARSS, p. 64, 2009.

[5] M. Villano, G. Krieger, and A. Moreira, "Staggered SAR: High-Resolution Wide-Swath Imaging by Continuous PRI Variation," IEEE Transactions on Geoscience and Remote Sensing, vol. 52, p. 4462, 2014.

[6] M. T. Wörle, C. Lenzen, T. Göttfert, A. Spörl, B. Grishechkin, F. Mrowka, M. Wickler, "The Incremental Planning System - GSOC’s Next Generation Mission Planning Framework," Space Ops, 2014.

[7] Kahle, R., Spiridonova, S. and Kirschner, M., "Improved Reference Orbits for the Repeat-Ground-Track Missions EnMAP and Tandem-L," Proceedings of the 26th International Symposium on Space Flight Dynamics, 2017.

[8] R. Kahle, S. D’Amico, "The TerraSAR-X Precise Orbit Control - Concept and Flight Results," Proceedings of the 24th International Symposium on Space Flight Dynamics, 2014.

[9] Krieger G., Hajnsek I., Papathanassiou K. P., Younis M., and Moreira A., "Interferometric Synthetic Aperture Radar (SAR) Missions Employing Formation Flying," Proceedings of the IEEE Vol. 98,No. 5,May 2010, 2010.

[10] M. Bachmann, D. B. Tridon, F. De Zan, G. Krieger, M. Zink, "Tandem-L Observation Concept - An Acquisition Scenario for the Global Scientific Mapping Machine," EUSAR, p. 1150, 2016.

[11] Schulze, D. Herman, J., Löw, S., "Formation Flight in Low-Earth-Orbit at 150 m Distance -AOCS In-Orbit Experience," Space Ops Conference, 2012.

[12] E. Maurer, R. Kahle, F. Mrowka, A. Ohndorf, S. Zimmermann, "Operational aspects of the TanDEM-X Science Phase," SpaceOps Conference, 2016.

[13] O. Montenbruck, M. Kirschner, S. D'Amico and S. Bettadpur, "E/I-Vector Separation for Safe Switching of the GRACE Formation," Aerospace Science and Technology, vol. 10, pp. 628-635, 2006.

[14] B. Schlepp, M. Kirschner, T. H. Sweetser, W. M. Klipstein, and S. Dubovitsky, "Flight Dynamics Challenges for the GRACE Follow-On Mission," 25th International Symposium on Space Flight Dynamics (ISSFD), 2015. 
[15] J. Herman, D. Fischer, D. Schulze, S. Löw, and M. Licht, "AOCS for TanDEM-X: Formation flight at 200m separation in low-Earth orbit," Space Ops Conference, 2010.

[16] D'Amico S. and Montenbruck O., "Proximity Operations of Formation-Flying Spacecraft using eccentricity/inclination vector separation," Journal of Guidance, Control, and Dynamics, 2005.

[17] Ardaens, J.-S., Kahle, R. Schulze, D., "In-flight performance validation of the TanDEM-X autonomous formation flying system," International Journal of Space Science and Engineering, 2013.

[18] Codazzi A., "Extended On-Call Support for TerraSAR-X," SpaceOps Conference, 2010.

[19] Satmon@Home, Heavens Above, [Online]. Available: http://www.heavens-above.com/.

[20] Arbinger, C. und D'Amico, S. und Feucht, U., "The GRACE Formation: Science Mode Pointing Performance Analysis," Third International Workshop on Satellite Constellations and Formation Flying, 2003. 